\title{
Loss of heterozygosity identifies genetic changes in chronic myeloid disorders, including myeloproliferative disorders, myelodysplastic syndromes and chronic myelomonocytic leukemia
}

\author{
Dennis P O’Malley ${ }^{1}$, Attilio Orazi ${ }^{2}$, Cherie H Dunphy ${ }^{3}$, Benjamin R Coleman ${ }^{4}$, \\ Rebecca A Marks ${ }^{4}$, Mingsheng Wang ${ }^{4}$ and Liang Cheng ${ }^{4}$ \\ ${ }^{1}$ US Labs, 2601 Campus Drive, Irvine, CA, USA; ${ }^{2}$ Division of Hematopathology, Department of Pathology and \\ Laboratory Medicine, Indiana University School of Medicine, Indianapolis, IN, USA; ${ }^{3}$ Department of \\ Pathology and Laboratory Medicine, University of North Carolina, Chapel Hill, NC, USA and ${ }^{4}$ Department of \\ Pathology and Laboratory Medicine, Indiana University School of Medicine, Indianapolis, IN, USA
}

\begin{abstract}
This study evaluates changes in genetic loci of chronic myeloid disorders using loss of heterozygosity (LOH) techniques. We present the combined results of three experiments. First, examination of a panel of genetic loci in groups of myeloproliferative disorders was evaluated. The second experiment involved microdissection of megakaryocytes from myeloproliferative disorders and comparison of their genetic changes to surrounding neoplastic marrow elements. Finally, we compared results of LOH studies of myeloproliferative disorders to those of myelodysplastic syndromes and chronic myelomonocytic leukemia. A total of 41 bone marrow biopsies were evaluated. Twenty-seven were myeloproliferative disorders (11 chronic idiopathic myelofibrosis, 11 essential thrombocythemia, 5 polycythemia vera). The remaining cases consisted of myelodysplastic syndromes (total $=5$; RAEB-1 $=2$; RAEB-2 $=2$; MDS, not otherwise specified $=1$ ) and chronic myelomonocytic leukemia $(n=8)$. The abnormalities in myeloproliferative disorders were distributed as follows: D7S2554-4/14 (5/14); D8S263-4/15 (5/15); D9S157-5/15 (5/15); D9S161-7/17 (6/17); D13S319-5/14 (4/14); TP53-5/16 (5/16); D20S108-4/15 (4/15). In 75\% cases diagnosed as essential thrombocythemia (6/8), both cases of polycythemia vera (2/2), and $29 \%$ cases of chronic idiopathic myelofibrosis $(2 / 7)$, there were genetic differences between the megakaryocytes and the surrounding marrow. These results suggest that in some cases, megakaryocytes have different clonal abnormalities than surrounding hematopoietic tissue. The genetic profiles of myeloproliferative disorders had several differences from those of myelodysplastic syndromes. Although different from both, chronic myelomonocytic leukemia appeared more similar to myeloproliferative disorders using these techniques.

Modern Pathology (2007) 20, 1166-1171; doi:10.1038/modpathol.3800951; published online 17 August 2007
\end{abstract}

Keywords: loss of heterozygosity; myeloproliferative disorders; myelodysplastic syndrome; myelodysplastic/ myeloproliferative diseases; acute myeloid leukemia

Myeloid disorders have a variety of underlying molecular and cytogenetic defects. Perhaps best described are those in chronic myeloproliferative disorders, including the $B C R / A B L$ translocation seen in chronic myelogenous leukemia and the

Correspondence: Dr DP O’Malley, MD, US Labs, 2601 Campus Drive, Irvine, CA 92612, USA.

E-mail: domalley@uslabs.net

Received 26 March 2007; revised and accepted 26 June 2007; published online 17 August 2007 recently described JAK2 mutation seen in a subset of other myeloproliferative disorders.

There are a wide variety of molecular and genetic techniques that are available for the analysis of myeloid disorders. However, some of these techniques are more difficult or impossible to apply to paraffin-embedded archival tissue. Loss of heterozygosity (LOH) analysis is a technique used to detect chromosomal imbalances. The basis of this testing is by comparing individual genetic loci from a specimen; in the typical circumstance, each parent would contribute distinct genes at a locus, which when 
hybridized with appropriate DNA probes, would be present as bands of two different sizes (eg, heterozygous). In many cancers, because of underlying defects, there is loss of one parental allele, which would present as $\mathrm{LOH}$ at the tested locus.

This technique has been applied to the study of acute myeloid leukemias and chronic myeloproliferative disorders, often with individual or only few probes evaluated. Several genetic loci have been identified as commonly involved. ${ }^{1-4}$ We developed a panel of seven chromosomal loci that have been shown to be frequently abnormal in myeloproliferative disorders. ${ }^{5}$ We then applied this panel of $\mathrm{LOH}$ probes in three experiments. First, we evaluated the occurrence and frequency of abnormalities in chronic myeloproliferative disorders. Subsequently, we microdissected megakaryocytes of chronic myeloproliferative disorders, and compared their genetic profiles to those of surrounding neoplastic hematopoietic tissues. In our final experiment, we compared the results of LOH studies in myeloproliferative disorders to those of a selection of myelodysplastic syndromes and chronic myelomonocytic leukemia, a hybrid myelodysplastic/ myeloproliferative disorder.

\section{Methods}

\section{Tissue Samples and Microdissection}

A combination of bone marrow core biopsies and clot sections were retrieved from the pathology files of Clarian Health Partners Department of Pathology and Laboratory Medicine (Indiana University School of Medicine; Riley Children's Hospital; Methodist Hospital, Indiana; Indianapolis, IN, USA) and University of North Carolina Hospitals, Division of Hematopathology, McLendon Clinical Laboratories (Chapel Hill, NC, USA). Specimens were obtained according to appropriate institutional policies. Cases with known diagnoses of myeloproliferative disorders, myelodysplastic syndromes, and chronic myelomonocytic leukemia were selected. Each diagnosis was reviewed to ensure compliance with current the 2001 WHO classification. In some cases, diagnoses were adjusted to correspond with the WHO classification.

Decalcification methods varied depending on institution (IU 10\% nitric acid; UNC 5\% acetic acid). Histologic sections were prepared from formalin-fixed, paraffin-embedded tissue and were stained with hematoxylin and eosin for microscopic evaluation. Tissue was removed from unstained sections and processed for $\mathrm{LOH}$ detection (see below). In each case of LOH analysis, a normal control tissue (eg, normal tissue from a surgical specimen from each patient) was used as a negative control.

In selected cases of myeloproliferative disorders, laser-assisted microdissection was performed using a PixCell II Laser Capture Microdissection (LCM) system (Arcturus Engineering, Mountain View, CA,
USA), as described previously. ${ }^{6}$ Briefly, the cells of interest are identified and thermoplastic transfer film is placed over the area of interest with the film on an uncoverslipped, uncharged glass slide. A laser is activated and passes through the transfer film. The transfer film rapidly heats and focally melts forming a matrix around the cells of interest as the polymer cools. The cell-film polymer is retracted from the surrounding tissue by lifting the cap. When all desired harvesting is complete, the cap and attached transfer film are removed from the setup and placed on a standard $0.5 \mathrm{ml}$ microcentrifuge tube containing a lysis buffer. The lysis buffer degrades the film on the underside of the cap and thereby releases a pure sample suspension with macromolecules (DNA, RNA, proteins) suitable for molecular analysis.

In these selected cases, at least 100 megakaryocytes were dissecting from the marrow. ${ }^{5}$ The surrounding hematopoietic tissue (without megakaryocytes) was then dissected from the marrow. After microdissection, the caps were digested by digested buffer containing $5 \mu \mathrm{g} / \mathrm{ml}$ of proteinase $\mathrm{K}$ at $37^{\circ} \mathrm{C}$ overnight. The samples are subjected to PCR after boiling for $10 \mathrm{~min}$. The results of megakaryocytic vs surrounding hematopoietic tissue were then compared.

\section{Detection of LOH}

Dissected tissues were deparaffinized with xylene and ethyl alcohol. Polymerase chain reaction (PCR) was used to amplify genomic DNA at seven specific loci on six different chromosomes: 7q31.1 (D7S2554); 8q24 (D8S263); 9p21 (D9S157, D9S161); 13q14 (D13S319); 17p13 (TP53); 20q12 (D20S108). ${ }^{6}$ PCR was performed in a $25 \mu$ l volume containing $0.8 \mu \mathrm{Ci}$ of $\alpha{ }^{32} \mathrm{P}$-labeled dATP, $2 \mu \mathrm{M}$ of mappair primers, $0.3 \mathrm{mM}$ of dNTP, $0.65 \mathrm{U}$ of iTaq DNA Polymerase (Bio-Rad Lab, Hercules, CA, USA). PCR amplification has an initial denaturation step of $95^{\circ} \mathrm{C}$ for $5 \mathrm{~min}$, followed by 35 cycles at $95^{\circ} \mathrm{C}$ for $30 \mathrm{~s}$, at $58^{\circ} \mathrm{C}$ for $30 \mathrm{~s}$, and at $72^{\circ} \mathrm{C}$ for $30 \mathrm{~s}$, and then followed by a single final extension step at $72^{\circ} \mathrm{C}$ for $7 \mathrm{~min}$. PCR products were diluted with stop buffer (0.04\% bromophenol blue, 0.04\% Xylene cyanol FF, $100 \%$ formamide). The samples were heated to $95^{\circ} \mathrm{C}$ for $5 \mathrm{~min}$ and then placed on ice. A $3 \mu \mathrm{l}$ volume of this mixture was loaded onto $6.5 \%$ polyacrylamide denaturing gel. Electrophoresis was performed at $80 \mathrm{~W}$ for $2 \mathrm{~h}$, followed by autoradiography with Kodak X-OMAT AR film (Eastman Kodak Company, Rochester, NY, USA) overnight.

A summary of these loci and their relation to important genes is presented in Table 1. The criterion for allelic loss was complete or nearly complete absence of one allele in tumor DNA. ${ }^{6}$ Polymerase chain reactions for each polymorphic microsatellite marker were repeated at least twice from the same DNA preparations and the same results were obtained. 


\section{Results}

A total of 41 bone marrow biopsies were evaluated. Twenty-seven with myeloproliferative disorders (11 chronic idiopathic myelofibrosis, 11 essential thrombocythemia, 5 polycythemia vera). The remaining cases consisted of myelodysplastic syndromes (total $=5 ;$ RAEB- $1=3 ;$ RAEB- $2=2$; MDS, not otherwise specified $=1$ ) and chronic myelomonocytic leukemia $(n=8)$. In analysis, we included an additional four bone marrow cases (3 chronic myelogenous leukemia, 1 chronic idiopathic myelofibrosis) from a previously published study. ${ }^{5}$ These four cases were not tested for the D20S108 locus.

Results of the LOH studies were considered informative if (1) two distinct bands were present in the control tissues (eg, heterozygous) and (2) either two (heterozygous) or one (homozygous) band(s) were seen on the test tissues. Of the 41 cases analyzed, 11 cases had one non-informative locus, 4 cases had two non-informative loci, and 1 case had three non-informative loci. No abnormal loci were identified in any of the negative control tissues.

\section{Myeloproliferative Disorders}

LOH analysis of chronic myeloproliferative disorders yielded informative results in all cases analyzed. All cases had one or more $\mathrm{LOH}$ abnormalities with the exception of one case each of essential thrombocythemia and polycythemia vera. The

Table 1 Summary of LOH loci tested

\begin{tabular}{lll}
\hline Chromosome & Locus & Gene \\
\hline 7q31.1 & D7S2554 & Putative TSG \\
8q24 & D8S263 & Telomeric to $M Y C$ \\
9p21 & D9S157 & Telomeric to p16 \\
9p21 & D9S161 & Centromeric to p16 \\
13q14 & D13S319 & Telomeric to $R B$ \\
17p13.1 & p53 & TP53 \\
20q12 & D20S108 & Telomeric to $C$-SRC \\
\hline
\end{tabular}

TSG, tumor suppressor gene. results of three cases of chronic myelogenous leukemia and one case of chronic idiopathic myelofibrosis from a previous study were added to analysis, as mentioned previously. All cases of chronic idiopathic myelofibrosis analyzed had 1-3 abnormal loci, essential thrombocythemia had 0-4 abnormalities, polycythemia vera had 0-2 abnormalities, and chronic myelogenous leukemia had one or two abnormalities, although this latter group was not tested for the D20S108 (as described previously). Frequencies of abnormalities for each locus are summarized in Table 2.

\section{Microdissection of Megakaryocytes}

LOH studies were performed on megakaryocytes that were dissected from 17 cases of myeloproliferative disorders ( 7 chronic idiopathic myelofibrosis, 8 essential thrombocythemia, 2 polycythemia vera). Each sample of tested megakaryocytes had at least one abnormal LOH locus. The abnormalities were distributed as follows in non-megakaryocytic hematopoietic tissue and megakaryocytes: D7S2554-4/14 (5/14); D8S263-4/15 (5/15); D9S157-5/15 (5/15); D9S161-7/17 (6/17); D13S319-5/14 (4/14); TP53$5 / 16(5 / 16)$; D20S108-4/15 (4/15). As in the original studies, no LOH was seen in the paired negative control tissues.

Comparisons of the neoplastic hematopoietic tissues to that of the microdissected megakaryocytes showed some striking differences. In $75 \%$ cases diagnosed as essential thrombocythemia (6/8), both cases of polycythemia vera $(2 / 2)$, and $29 \%$ cases of chronic idiopathic myelofibrosis (2/7), there were LOH differences identified between the megakaryocytes and the surrounding marrow (Table 3). Further, two cases of essential thrombocythemia (case 1, case 4) showed distinct differences between all abnormal loci in the megakaryocytes vs those of the surrounding marrow. Notably, genetic differences between megakaryocytes and surround neoplastic hematopoietic tissues were particularly frequent in essential thrombocythemia at D8S263 $(3 / 7,43 \%), \quad$ D13S319 (3/7, 43\%), and TP53 $(5 / 8,63 \%)$.

Table 2 Summary of results of loss of heterozygosity studies

\begin{tabular}{|c|c|c|c|c|c|c|c|}
\hline & D7S2554 & D8S263 & D9S157 & D9S161 & $D 13 S 319$ & TP53 & D20S108 \\
\hline CIMF & $27 \%(3 / 11)$ & $50 \%(5 / 10)$ & $27 \%(3 / 11)$ & $18 \%(2 / 11)$ & $36 \%(4 / 11)$ & $25 \%(3 / 12)$ & $30 \%(3 / 10)$ \\
\hline $\mathrm{ET}$ & $30 \%(3 / 10)$ & $18 \%(2 / 11)$ & $36 \%(4 / 11)$ & $27 \%(3 / 11)$ & $30 \%(3 / 10)$ & $27 \%(3 / 11)$ & $10 \%(1 / 10)$ \\
\hline PV & $25 \%(1 / 4)$ & $0 \%(0 / 4)$ & $0 \%(0 / 4)$ & $40 \%(2 / 5)$ & $0 \%(0 / 3)$ & $25 \%(1 / 4)$ & $40 \%(2 / 5)$ \\
\hline CML & $0 \%(0 / 3)$ & $50 \%(1 / 2)$ & $0 \%(0 / 2)$ & $50 \%(1 / 2)$ & $0 \%(0 / 3)$ & $25 \%(1 / 4)$ & NP \\
\hline$A L L C M P D$ & $25 \%(7 / 28)$ & $30 \%(8 / 27)$ & $25 \%(7 / 28)$ & $28 \%(8 / 29)$ & $26 \%(7 / 27)$ & $26 \%(8 / 31)$ & $28 \%(7 / 25)$ \\
\hline MDS & $50 \%(3 / 6)$ & $0 \%(0 / 5)$ & $0 \%(0 / 6)$ & $33 \%(2 / 6)$ & $17 \%(1 / 6)$ & $33 \%(2 / 6)$ & $17 \%(1 / 6)$ \\
\hline CMML & $25 \%(2 / 8)$ & $43 \%(3 / 7)$ & $38 \%(3 / 8)$ & $25 \%(2 / 8)$ & $50 \%(4 / 8)$ & $43 \%(3 / 7)$ & $29 \%(2 / 7)$ \\
\hline
\end{tabular}

CIMF, chronic idiopathic myelofibrosis; CML, chronic myelogenous leukemia; CMML, chronic myelomonocytic leukemia; CMPD, chronic myeloproliferative disorder; ET, essential thrombocythemia; MDS, myelodysplastic syndrome; NP, not performed; PV, polycythemia vera. 
Table 3 Loss of heterozygosity results in 17 myeloproliferative bone marrows

$\begin{array}{lllllll}\text { D7S2554 } & \text { D8S263 } & \text { D9S157 } & \text { D9S161 } & \text { D13S319 } & \text { TP53 } & \text { D20S108 }\end{array}$

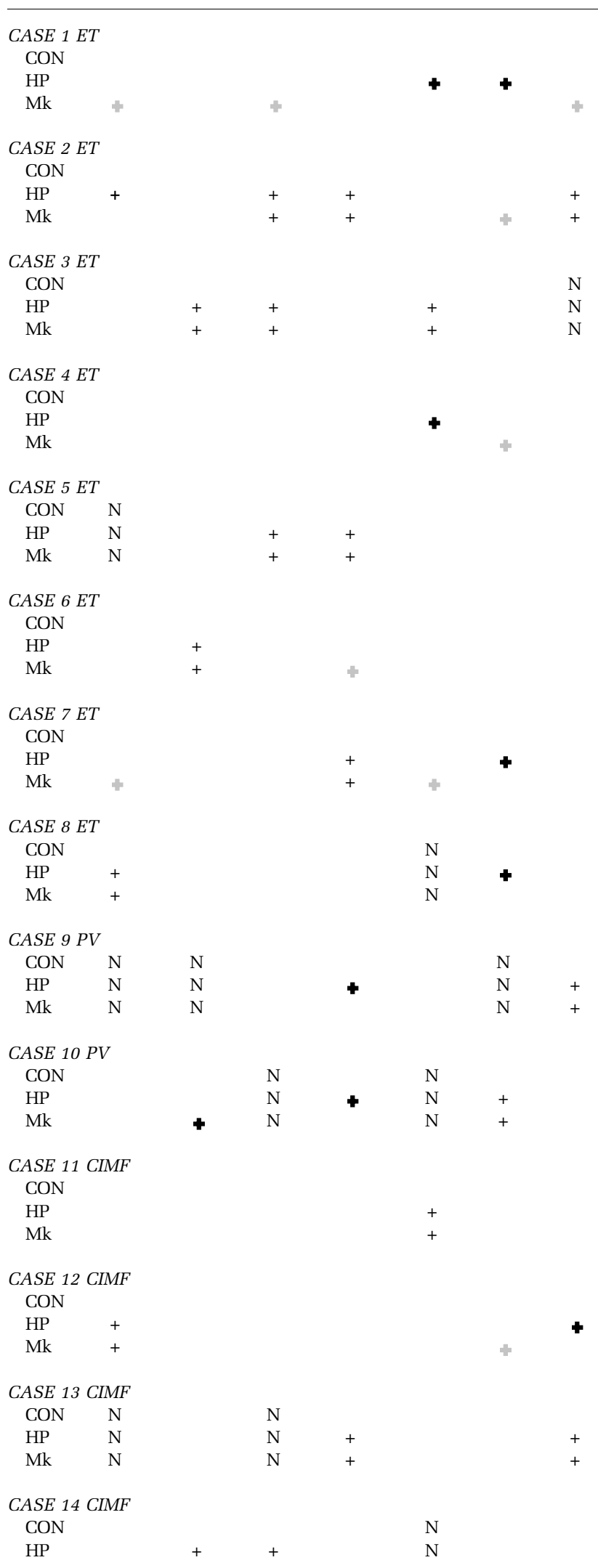

Table 3 Continued

\begin{tabular}{|c|c|c|c|c|c|c|c|}
\hline & D7S2554 & D8S263 & D9S157 & D9S161 & D13S319 & TP53 & D20S108 \\
\hline $\mathrm{Mk}$ & & + & + & & $\mathrm{N}$ & & \\
\hline \multicolumn{8}{|c|}{ CASE 15 CIMF } \\
\hline HP & & & & + & & + & \\
\hline Mk & & & & + & & + & \\
\hline \multicolumn{8}{|c|}{ CASE 16 CIMF } \\
\hline CON & & $\mathrm{N}$ & & & & & $\mathrm{N}$ \\
\hline $\mathrm{HP}$ & + & $\mathrm{N}$ & + & & + & & $\mathrm{N}$ \\
\hline Mk & + & $\mathrm{N}$ & & & + & & $\mathrm{N}$ \\
\hline \multicolumn{8}{|c|}{ CASE 17 CIMF } \\
\hline $\mathrm{HP}$ & & + & & & & & \\
\hline Mk & & + & & & & & \\
\hline
\end{tabular}

CIMF, chronic idiopathic myelofibrosis; CML, chronic myelogenous leukemia; CON, non-hematopoietic normal tissue controls; ET, essential thrombocythemia; HP, non-megakaryocytic hematopoietic cells; Mk, megakaryocytes; PV, polycythemia vera.

'+' indicates presence of abnormal, homozygous signal (eg, loss of heterozygosity). ' $\mathrm{N}$ ' indicates non-informative study. Gray '", indicates clonal difference in megakaryocytic lineage, not seen in surrounding hematopoietic tissue. Bold '†' indicates clonal difference in non-megakaryocytic cells, not seen in corresponding megakaryocytic lineage.

\section{Comparison of Myeloid Disorders}

$\mathrm{LOH}$ analysis was performed in chronic myelomonocytic leukemia $(n=8)$ and myelodysplastic syndromes $(n=5)$ and compared to the results seen in myeloproliferative disorders (Table 2). In chronic myelomonocytic leukemia, 7/8 (88\%) cases evaluated had at least one or more abnormal LOH locus (Table 2). All cases of myelodysplastic syndromes $(5 / 5)$ had one or more $\mathrm{LOH}$ abnormality.

Chronic myelomonocytic leukemia had more abnormalities per case than seen in myelodysplastic syndromes or chronic myeloproliferative disorders (see Table 4). The patterns of abnormalities in chronic myelomonocytic leukemia appear more similar to those of myeloproliferative disorders. However, the frequency of abnormalities was increased in four loci (D8S263, D9S157, D13S319, TP53) in chronic myelomonocytic leukemia. The pattern of abnormalities in myelodysplastic syndromes showed an increased frequency of abnormalities in D7S2554 and no abnormalities in D8S263 and D9S157, in contrast to the findings in chronic myeloproliferative disorders and chronic myelomonocytic leukemia.

\section{Conclusions}

Our results show that evaluation of LOH of myeloid disorders, specifically chronic myeloproliferative disorders, can be used as a tool to evaluate the underlying genetic abnormalities in myeloid 
Table 4 Average number of abnormal genetic loci in chronic myeloid disorders

\begin{tabular}{lll}
\hline & & $\begin{array}{c}\text { Average number of } \\
\text { abnormal loci per case } \\
\text { (out of } 7 \text { loci) }\end{array}$ \\
\hline All MPD $(n=31)$ & CIMF $(n=12)$ & 1.71 \\
& ET $(n=11)$ & 1.9 \\
& PV $(n=5)$ & 1.8 \\
MDS $(n=6)$ & CML $(n=3)^{\mathrm{a}}$ & 1.2 \\
CMML $(n=8)$ & & 1.33 \\
& & 1.5 \\
& & 2.38
\end{tabular}

CIMF, chronic idiopathic myelofibrosis; CML, chronic myelogenous leukemia; CMML, chronic myelomonocytic leukemia; ET, essential thrombocythemia; MDS, myelodysplastic syndrome; MPD, myeloproliferative disorder; PV, polycythemia vera.

${ }^{\mathrm{a}}$ Cases included from previous study for analysis. Locus D20S108 was not tested in these specimens.

disorders. As in our previous studies, the panel of genetic loci developed (Table 1) identifies frequent abnormalities in the large majority of cases tested. The results of our first experiment highlighted the genetic heterogeneity of the $B C R / A B L$-negative myeloproliferative disorders. No trends or distinctive abnormalities were readily apparent among specific diagnoses that would suggest that these groups had unique or characteristic genetic abnormalities using this LOH panel.

These results of our second study provide supportive evidence for clonal evolution in different cell lineages within some myeloproliferative disorders. This phenomenon has been described in several other types of malignancies, including melanoma and carcinomas. ${ }^{7-9}$ Based on the comparison of $\mathrm{LOH}$ in megakaryocytes and surround neoplastic myeloid tissue, it appears that in a subset of cases, different cell lineages have different genetic abnormalities. The underlying abnormalities of a neoplastic stem cell (such as $J A K 2$ or $B C R / A B L$ ) acquire additional genetic abnormalities and possibly have different differentiation or maturation signals to develop into specific lineages. Further, this may suggest that some lineages may be more susceptible to acquiring additional abnormalities, and that genetic evolution and possibly disease acceleration or leukemic transformation may arise from these 'susceptible' lineages.

Issues of tissue contamination could be raised to question the results of the megakaryocytic microdissection studies. However, if there was contamination, the results would then be the same for both megakaryocytes and surrounding hematopoietic tissue. The lack of those similarities in several cases and the presence of distinct differences in a subset of cases suggest that the contamination of the megakaryocyte test tissues by the surrounding hematopoietic tissue likely did not occur. Further, the presence of no abnormalities in any control tissue, compared to the presence of abnormalities in all cases (with the exclusion of non-informative loci) suggests that the results are valid.

Our final experiment, comparing the genetic loci in chronic myeloproliferative disorders, chronic myelomonocytic leukemia, and myelodysplastic syndromes also provided interesting results. The question of whether chronic myelomonocytic leukemia was more like a myeloproliferative disorder or a myelodysplastic syndrome has been present since before the French-American-British (FAB) classification of myeloid disorders. Our results seem to suggest that based on this limited selection of genetic loci, chronic myelomonocytic leukemia is more similar to chronic myeloproliferative disorders, rather than the myelodysplastic syndromes tested.

Evaluation of $J A K 2$ loci were not undertaken in this study. JAK2 mutations have recently been identified as being a fundamental genetic abnormality in many non-CML chronic myeloproliferative disorders. ${ }^{10,11}$ Our results suggest that although not necessarily causative of disease, several other genetic loci are frequently involved in chronic myeloproliferative disorders.

These results are intriguing and show similar findings to those of our previous study on splenic tissue with myeloproliferative disorders. ${ }^{5}$ These studies support the value of $\mathrm{LOH}$ as a robust technique for evaluating the genetic changes that are present in a variety of bone marrow disorders. In addition, our results emphasize that there is considerable genetic heterogeneity within the several groups of myeloid disorders studied. Whereas there may be fundamental defects that are the primary cause of myeloid disorders (such as $B C R / A B L$ or $J A K 2$ mutations), there are frequently additional genetic abnormalities that may account for clinical differences in individual cases. Further, evaluation of the nature of clonal evolution in distinct lineages of hematopoietic cells in myeloid disorders using powerful techniques such as LOH studies suggests future avenues of research with possible important clinical implications.

\section{Acknowledgement}

Portions of these results were presented in abstract form at the 2004, 2005 and 2006 annual United States and Canadian Academy of Pathology annual meetings.

\section{References}

1 Tripputi P, Cassani B, Alfano R, et al. Chromosome 7 monosomy and deletions in myeloproliferative diseases. Leuk Res 2001;25:735-739.

2 Reeder TL, Bailey RJ, Dewald GW, et al. Both B and T lymphocytes may be clonally involved in myelofibrosis with myeloid metaplasia. Blood 2003;101: 1981-1983. 
3 Gribble SM, Reid AG, Roberts I, et al. Genomic imbalances in CML blast crisis: 8q24.12-q24.13 segment identified as a common region of over-representation. Genes Chromosom Cancer 2003;37:346-358.

4 Kralovics R, Buser AS, Teo SS, et al. Comparison of molecular markers in a cohort of patients with chronic myeloproliferative disorders. Blood 2003;102: 1869-1871.

5 O'Malley DP, Orazi A, Wang M, et al. Analysis of loss of heterozygosity and X-chromosome inactivation in spleens with myeloproliferative disorders and acute myeloid leukemia. Mod Pathol 2005;18: 1562-1568.

6 Katona TM, Jones TD, Wang M, et al. Molecular evidence for independent origin of multifocal neuroendocrine tumors of the enteropancreatic axis. Cancer Res 2006;66:4936-4942.
7 Sung MT, Wang M, Maclennan G, et al. Histogenesis of sarcomatoid urothelial carcinoma of the urinary bladder: evidence for a common clonal origin with divergent differentiation. J Pathol 2007;211:420-430.

8 Fujii H, Ajioka Y, Kazami S, et al. Loss of heterozygosity in the clonal evolution of flat colorectal neoplasms. J Pathol 2002;197:298-306.

9 Katona TM, Jones TD, Wang $\mathrm{M}$, et al. Genetically heterogeneous and clonally unrelated metastases may arise in patients with cutaneous melanoma [abstract]. Mod Pathol 2007;20(Suppl 2):94A.

10 Frohling S, Scholl C, Gilliland DG, et al. Genetics of myeloid malignancies: pathogenetic and clinical implications. J Clin Oncol 2005;23:6285-6295.

11 Tefferi A, Barbui T. Bcr/abl-negative, classic myeloproliferative disorders: diagnosis and treatment. Mayo Clin Proc 2005;80:1220-1232. 